\title{
Fatores determinantes de perda de peso em adultos submetidos a intervenções dietoterápicas
}

\author{
Determinant factors associated with weight \\ loss in adults on diet interventions
}

Luciana Verçoza Viana', Tatiana Pedroso de Paula', Cristiane

Bauermann Leitão', Mirela Jobim Azevedo'

\begin{abstract}
RESUMO
Objetivo: Analisar os fatores determinantes da perda de peso ( $\geq 5 \%$ ) resultante de atendimento ambulatorial individual. Sujeitos e métodos: Estudo de coorte retrospectivo com 318 pacientes com sobrepeso/obesidade em atendimento individual para perder peso. Resultados: Quinze por cento dos pacientes não perderam peso, 35,1\% apresentaram perda $<5 \%, 35,4 \%$ entre $5-10 \%$ e $13,9 \%$ perda $\geq 10 \%$. Os pacientes que perderam $\geq 5 \%$ de peso $(-7,6 \pm 3,3 \mathrm{~kg} ; \mathrm{n}=156)$ tiveram maior número e menor intervalo entre consultas e maior frequência de atendimentos particulares, acompanhamento por nutricionista e atendimentos multidisciplinares. Ainda, houve neste grupo menor prescrição de medicamentos antiobesidade e o valor calórico total da dieta foi maior do que nos demais pacientes. Em análises multivariadas de regressão de Cox apenas o intervalo entre consultas e o número total de consultas permaneceram inversamente associados à perda de peso. Conclusões: Os determinantes de perda de peso $\geq 5 \%$ foram um menor número de consultas com um menor intervalo entre os atendimentos. Arq Bras Endocrinol Metab. 2013;57(9):717-21
\end{abstract}

\section{Descritores}

Determinantes; perda de peso; obesidade

\begin{abstract}
Objective: To analyze the determinants for weight loss ( $\geq 5 \%$ ) resulting from outpatient individual appointments. Subjects and methods: A retrospective cohort study was conduct in 318 overweight/obese patients seeking individual care to lose weight. Results: Fifteen percent of the patients did not lose weight; $35.1 \%$ had lost < 5\%; $35.4 \%$ had lost between 5 and $10 \%$; and $13.9 \%$ had lost $\geq 10 \%$. Patients who lost $\geq 5 \%$ body weight $(-7.6 \pm 3.3 \mathrm{~kg}, \mathrm{n}=156)$ had a greater number of visits at a shorter interval, and greater frequency of visits with a registered dietitian and multidisciplinary care. This group had a lower prescription of anti-obesity drugs and their total calorie intake was higher than the other patients. In multivariate Cox regression, only the interval between appointments and the total number of visits remained inversely associated with weight loss. Conclusions: The determinants of $\geq 5 \%$ weight loss were fewer visits with a shorter interval between appointments. Arq Bras Endocrinol Metab. 2013;57(9):717-21
\end{abstract}

\section{Keywords}

Determinants; weight loss; obesity

\section{INTRODUÇÃO}

$\mathrm{O}$ excesso de peso e a obesidade vêm aumentando de forma preocupante em termos de saúde pública no Brasil. Segundo a Pesquisa de Orçamento Familiar (POF 2008-2009) publicada em 2010, de 1974-1975 a 2008-2009 a prevalência de excesso de peso em adultos aumentou em quase três vezes no sexo masculino (de $18,5 \%$ para $50,1 \%$ ) e em quase duas vezes no sexo feminino (de $28,7 \%$ para $48,0 \%$ ) (1). No
1 Departamento de Endocrinologia, Hospital de Clínicas de Porto Alegre (HCPA), Porto Alegre, RS, Brazil
Correspondência para:

Luciana Verçoza Viana

Departamento de Endocrinologia, Hospital de Clínicas de Porto Alegre Rua Ramiro Barcelos, 2350,

prédio $12,4^{\circ}$ andar

90035-003 - Porto Alegre, RS, Brasil vercoza@yahoo.com

Recebido em 16/Abr/2013 Aceito em 25/Jul/2013 mesmo período, a prevalência de obesidade aumentou de $2,8 \%$ para $12,4 \%$ nos homens e de $8,0 \%$ para $16,9 \%$ nas mulheres (1). Nos Estados Unidos, o "Centers for Disease Control and Prevention" (CDC) estima que um terço da população adulta americana seja obesa (2).

Obesidade e sobrepeso estão associados a uma série de comorbidades, tais como diabetes melito tipo 2, apneia do sono, dislipidemia, hipertensão arterial sistêmica (HAS), osteoartrite e câncer (3). O índice de massa cor- 
poral (IMC) está associado com a mortalidade cardiovascular tanto em homens quanto em mulheres $(4,5)$. Por outro lado, a perda intencional de peso está associada à melhora de muitas dessas complicações. A Associação Americana de Diabetes (ADA) recomenda um programa de perda de $7 \%$ do peso aliada a 150 minutos de atividade física moderada semanal ou 75 minutos de atividade vigorosa por semana para redução do risco de diabetes em pacientes com pré-diabetes e sobrepeso (6). $\mathrm{O}$ "Seventh Report of the Joint National Committee on Prevention, Detection, Evaluation, and Treatment of High Blood Pressure" (JNC7) considera que a perda de peso de cerca de 4,5 kg é capaz de reduzir ou prevenir hipertensão arterial em indivíduos com sobrepeso (7).

Inúmeras são as estratégias para a redução de peso. Entretanto, a perda ponderal e a manutenção desta em longo prazo representam na prática clínica um problema de difícil manejo. A redução calórica, independente da composição de macronutrientes da dieta, parece ser o fator mais importante para a diminuição de peso (8-10). Há evidências de que o uso de medicamentos antiobesidade, associado a programas de redução calórica e atividade física, aumente ainda mais a perda de peso (11).

A abordagem multidisciplinar para perda de peso parece ser melhor do que a individual (12-14). Também estratégias terapêuticas dietoterápicas em grupo parecem ser mais efetivas no manejo da obesidade (15). Entretanto, frequentemente esses tipos de abordagem, tanto do ponto de vista de organização governamental quanto do ponto de vista econômico individual, são de difícil implementação. Na prática clínica diária um grande número de pacientes acaba procurando atendimento em consultórios ou clínicas privadas, com atendimento feito por um único profissional. Ainda, nesse contexto de atendimento individual para perda de peso voluntária não são plenamente estabelecidos os fatores associados à perda de peso.

\section{OBJETIVOS}

Analisar os fatores determinantes de perda de peso voluntária resultante de atendimento ambulatorial individual.

\section{SUJEITOS E MÉTODOS}

Este estudo de coorte retrospectivo avaliou pacientes maiores do que 18 anos de idade com IMC $>25 \mathrm{~kg} / \mathrm{m}^{2}$, com pelo menos três consultas para avaliação do peso e cujo motivo principal da consulta foi o desejo de perder peso. Trata-se de uma amostra randômica selecionada em consultório de médico endocrinologista e nutricionista, identificada por meio do programa random.org. O principal desfecho foi perda de peso $\geq 5 \%$ ao final do período de acompanhamento. As variáveis em estudo foram: características dos pacientes [idade, sexo, IMC, nível educacional ( $>8$ anos de estudo), tabagismo ativo, atividade física, comorbidades, medicamentos em uso]; tentativas prévias de emagrecimento; intervenções farmacológicas antiobesidade associadas (anorexígenos, moderadores de apetite) e características do atendimento [tipo de consulta (convênio, privada), número de consultas, intervalo entre as consultas, tempo de acompanhamento]. Foram excluídos pacientes em avaliação para cirurgia bariátrica, com doença psiquiátrica, gestantes, em uso de medicamentos relacionados ao aumento de peso e/ou apetite (por exemplo, glicocorticoides).

Dois consultórios de atendimento privado, um de endocrinologista (LVV) e outro de nutricionista (TPP), localizados na área central de Porto Alegre/RS, foram selecionados por conveniência. Ambas as profissionais tinham cinco anos de formadas e atuavam em consultório próprio, tendo experiência clínica em tratamento para emagrecimento de pacientes com sobrepeso ou obesidade.

As medidas antropométricas utilizadas para a avaliação do estado nutricional dos pacientes foram: peso $(\mathrm{kg})$ com roupas leves e sem sapatos (balança antropométrica), estatura $(\mathrm{m})$, cálculo do IMC [peso $(\mathrm{kg}) /$ altura $^{2}(\mathrm{~m})$ ]. Foi considerado fisicamente ativo qualquer indivíduo que referisse prática de atividade física regular (pelo menos duas vezes na semana). Tabagismo foi definido como uso atual de tabaco.

As intervenções dietoterápicas foram: dieta padrão, prescrita pelo endocrinologista e dieta calculada, prescrita pela nutricionista. $\mathrm{Na}$ dieta padrão, os pacientes foram orientados a adotar dieta hipocalórica com valor calórico total (VCT) de $1.400 \mathrm{kcal} /$ dia (25\% proteína, 25\% lipídios, $49 \%$ carboidratos - 38 g de fibra) para mulheres e $1.800 \mathrm{kcal} /$ dia para homens $(24 \%$ proteínas, $21 \%$ lipídios, $55 \%$ carboidratos). A dieta calculada foi prescrita com um VCT de $20 \mathrm{kcal} / \mathrm{kg} / \mathrm{dia}$ (com 2025\% proteína, 25-30\% lipídios, 50-55\% carboidratos, $14 \mathrm{~g} /$ fibras para cada $1.000 / \mathrm{kcal}$ consumidas).

Para o cálculo do tamanho amostral, levaram-se em conta estudos realizados em atenção primária que compararam a perda de peso obtida quando o tratamento foi realizado por médicos generalistas e nutricionistas $(14,16)$. Utilizando um valor de perda ponderal de 5 
a $10 \%$, em análise de não inferioridade, estimou-se que um total de 312 pacientes seria necessário para obter um poder de $80 \%$ com um alfa de 0,05 .

Para comparação das características basais dos pacientes, foram utilizados testes $t$ de Student e qui-quadrado. Análises multivariadas de regressão de Cox foram realizadas para estimar a chance de perda de peso de 5 e $10 \%$ (variáveis dependentes) de acordo com as características dos pacientes (variáveis independentes) que atingiram ou não os desfechos de interesse. As variáveis independentes foram incluídas nos modelos de acordo com a significância na análise univariada. Os dados foram apresentados como média \pm DP, mediana (intervalo interquartil 25-75) ou número de casos (\%). Valores de $\mathrm{P}<0,05$ foram considerados estatisticamente significativos. Todas as análises foram realizadas utilizando o programa SPSS (versão 18.0; SPSS, Inc. Chicago, IL).

\section{RESULTADOS}

\section{Características gerais da amostra}

Dos 769 pacientes selecionados (endocrinologista, $\mathrm{n}$ = 486; nutricionista, $\mathrm{n}=283$ ), o principal motivo de exclusão do estudo (cerca de $60 \%$ ) foi o não compa- recimento a pelo menos três consultas. Portanto, 318 pacientes foram incluídos no estudo, sendo $51 \%$ provenientes do consultório do endocrinologista e $49 \%$, da nutricionista.

Entre os pacientes incluídos no estudo (Tabela 1), a idade média foi de 38 anos e o IMC inicial, de 31 $\mathrm{kg} / \mathrm{m}^{2}$ (obesidade grau I), com predomínio do sexo feminino $(72,3 \%)$. Mais da metade dos pacientes apresentavam tentativas prévias de perda de peso; $38 \%$ desses haviam feito uso prévio de medicamentos para emagrecer e $40 \%$ dos pacientes tinham comorbidades associadas (20\% HAS, 17\% dislipidemia, 12\% diabetes ou pré-diabetes). A média de consultas foi de 6,0 nos 8,0 meses de acompanhamento, perfazendo um intervalo de 1,3 mês entre as consultas. Quanto ao tipo de consulta, $22 \%$ dos pacientes faziam o tratamento particular e os demais utilizavam convênios. Trinta e três por cento dos pacientes utilizaram medicação para emagrecimento durante o estudo, sendo $60 \%$ desses pacientes acompanhados por endocrinologista e $5 \%$, pela nutricionista. A sibutramina foi a medicação utilizada em $84 \%$ dos casos. A perda média de peso durante o estudo foi $4,4 \mathrm{~kg}$, com um valor de perda máximo de $5,8 \pm 3,8 \mathrm{~kg}$ ao redor do $5^{\circ}$ mês de acompanhamento.

Tabela 1. Características clínicas dos 318 pacientes estudados, respondedores e não respondedores

\begin{tabular}{|c|c|c|c|c|}
\hline & Total & Respondedores & Não respondedores & $\mathbf{p}$ \\
\hline$n$ & 318 & 156 & 162 & \\
\hline Idade (anos) & $37,5 \pm 12,7$ & $37 \pm 12$ & $38 \pm 13$ & 0,480 \\
\hline Sexo masculino & 89 (28\%) & $50(32 \%)$ & $39(24 \%)$ & 0,134 \\
\hline Consulta particular & $72(22 \%)$ & $51(35 \%)$ & $21(14 \%)$ & $<0,01$ \\
\hline Ensino superior completo & $74(23 \%)$ & $43(28 \%)$ & $31(26 \%)$ & 0,134 \\
\hline Atendimento por nutricionista & $154(48 \%)$ & $101(65 \%)$ & $53(33 \%)$ & $<0,01$ \\
\hline Tentativa prévia de perda de peso & $176(55 \%)$ & $50(32 \%)$ & $39(24 \%)$ & 0,067 \\
\hline Comorbidades & $128(40 \%)$ & $71(46 \%)$ & $85(54 \%)$ & 0,068 \\
\hline Acompanhamento multidisciplinar & $84(26,4 \%)$ & $50(32 \%)$ & $34(23 \%)$ & 0,038 \\
\hline Atividade física & $111(35 \%)$ & $54(38 \%)$ & 57 (38\%) & 1,000 \\
\hline Tabagismo & $41(13 \%)$ & $25(16 \%)$ & $13(13 \%)$ & 0,591 \\
\hline Uso álcool & $130(41 \%)$ & $72(46)$ & $53(49 \%)$ & 0,619 \\
\hline IMC inicial (kg/m²) & $31 \pm 4$ & $31 \pm 4,1$ & $31 \pm 4,3$ & 0,845 \\
\hline Medicamento antiobesidade & $104(33 \%)$ & $40(26 \%)$ & $64(40 \%)$ & 0,009 \\
\hline$N^{0}$ de consultas & $5,5 \pm 3$ & $5,9 \pm 2,6$ & $5,3 \pm 3,2$ & 0,042 \\
\hline Tempo de acompanhamento (meses) & $8 \pm 8,2$ & $7,22 \pm 6,7$ & $8,6 \pm 9,4$ & 0,149 \\
\hline Intervalo entre consultas (meses) & $1,3 \pm 1$ & $1,15 \pm 0,63$ & $1,50 \pm 1,16$ & 0,002 \\
\hline VCT (kcal/kg/dia) & $18,7 \pm 2,4$ & $19,0 \pm 2,0$ & $18,3 \pm 2,7$ & 0,003 \\
\hline Variação peso (kg) & $-4,35 \pm 4,35$ & $-7,6 \pm 3,3$ & $-1,2 \pm 2,63$ & --- \\
\hline
\end{tabular}

Respondedores definidos com perda de peso final $\geq 5 \%$; IMC: índice de massa corporal; VCT: valor calórico total. 
Ao final do acompanhamento, $15,5 \%$ dos pacientes não apresentaram perda de peso, $35,1 \%$ apresentaram perda de peso inferior a $5 \%, 35,4 \%$ perda entre 5 - $10 \%$ e $13,9 \%$ dos pacientes obtiveram perda $\geq 10 \%$. Foi considerada clinicamente relevante perda de peso final $\geq 5 \%$.

\section{Comparação entre pacientes respondedores e não respondedores}

A tabela 1 mostra as características basais dos pacientes respondedores (perda de peso $\geq 5 \%$ ) e dos não respondedores ao tratamento para redução de peso ao final do acompanhamento. No grupo dos respondedores, houve um maior número de consultas particulares e acompanhamento por nutricionista, mais atendimentos multidisciplinares, maior número total de consultas e menor intervalo entre as consultas. Neste grupo, houve menor frequência de uso de medicamentos antiobesidade e o valor calórico prescrito das dietas foi maior quando comparado aos não respondedores.

\section{Fatores determinantes da perda de peso: análise multivariada de Cox}

$\mathrm{Na}$ análise multivariada de regressão de Cox, perda ponderal $>5 \%$ foi definida como variável dependente (Tabela 2). Foram incluídos no modelo (variáveis independentes): tipo de consulta (particular ou convênio), profissional que realizou o atendimento (endocrinologista ou nutricionista), atendimento multidisciplinar, uso de medicação para emagrecer, VCT da dieta, número de consultas e intervalo entre as consultas. Foram determinantes da perda de peso o intervalo (meses) entre as consultas $(\mathrm{OR}=0,002 ; 95 \%$ IC 0,001-0,010; $\mathrm{P}<0,001) \mathrm{e}$ o número total de consultas (OR 0,310; $95 \%$ IC 0,228 $0,421 ; \mathrm{P}<0,001)$. Aplicando o mesmo modelo para perda $\geq 10 \%$, permaneceram também como determinantes o intervalo entre consultas $(\mathrm{OR}=0,003 ; 95 \% \mathrm{IC}$ 0,000-0,041; $\mathrm{P}<0,001)$ e o número total de consultas $(\mathrm{OR}=0,466 ; 95 \%$ IC 0,299-0,724; $\mathrm{P}<0,001)$.

Tabela 2. Determinantes de perda de peso $>5 \%$ : modelo multivariado de regressão de Cox

\begin{tabular}{lccc}
\hline Variáveis independentes & HR & IC 95\% & P \\
\hline Acompanhamento com nutricionista & 1,776 & $0,814-3,873$ & 0,822 \\
Consulta particular & 0,941 & $0,552-1,602$ & 0,822 \\
Acompanhamento multidisciplinar & 1,419 & $0,814-3,873$ & 0,186 \\
VCT (kcal/kg/dia) & 1,000 & $0,889-1,125$ & 0,997 \\
Intervalo entre consultas (meses) & 0,002 & $0,001-0,010$ & $<0,01$ \\
Número de consultas & 0,310 & $0,228-0,421$ & $<0,01$ \\
\hline
\end{tabular}

\section{DISCUSSÃO}

O presente estudo apresenta uma análise de estratégias ambientadas em um cenário de vida real: o consultório privado de profissionais da saúde que trabalham com sobrepeso/obesidade. Nesta amostra, constituída predominantemente de mulheres com obesidade grau I e idade média de 38 anos, houve diminuição de cerca de $4 \mathrm{~kg}(5,6 \%)$ ao longo de oito meses de acompanhamento. Uma perda de peso $\geq 5 \%$ foi obtida em cerca de $50 \%$ dos pacientes e somente $14 \%$ obtiveram perda $\geq$ $10 \%$. O atendimento por endocrinologista ou nutricionista ou uso de medicamentos antiobesidade não foram determinantes da perda de peso. Em análise multivariada, demonstramos que o menor número e o menor intervalo entre as consultas foram associados à perda de peso final $\geq 5$ ou $10 \%$. Demonstramos que a razão de azares para essa perda de peso é de 0,002 para cada mês adicional entre as consultas, ou seja, consultar para emagrecer com um intervalo de dois meses, em vez de um mês, reduz a chance de perda de peso em quase $100 \%(99,8 \%)$. Já a razão de azares para emagrecimento de 0,3 para o número de consultas sugere que a perda de peso reduza ao longo das consultas. De fato observamos que a maior perda ponderal ocorre nas primeiras consultas (perda máxima no quinto mês). Esses achados indicam que o mais importante determinante da perda de peso voluntária é o intervalo entre consultas: quanto menor tempo entre as consultas, maior a chance de perda de peso.

O tratamento da obesidade em consultórios privados diferencia-se de outros atendimentos, pois nestes o paciente procura a consulta espontaneamente. Também até o presente momento não existiam dados comparativos dos resultados de perda de peso quando o tratamento foi realizado por diferentes especialidades que trabalham de forma individual com obesidade. A maioria dos estudos em sobrepeso ou obesidade foi realizada em ambientes com atendimento multidisciplinar, muitas vezes envolvendo incentivos aos participantes e limitando a inclusão de pacientes devido aos inúmeros critérios de seleção.

A perda de peso obtida neste estudo foi similar à observada em ensaios clínicos randomizados. Em estudo realizado com adultos obesos e pelo menos um fator de risco cardiovascular, foi demonstrada uma perda de peso entre 5 e $6 \mathrm{~kg}$ no grupo intervenção comparado ao grupo placebo, ao longo dos 24 meses (16). Em uma revisão sistemática com metanálise de estudos so- 
bre emagrecimento com no mínimo um ano de seguimento, a perda de peso foi de 5 a $8,5 \%$ nos primeiros seis meses, com um platô de peso a partir de então (17). Esses dados estão em concordância com os do presente estudo, no qual a maior perda ponderal foi observada aos cinco meses.

Finkler e cols. (18), em estudo de metanálise, buscaram estabelecer fatores relacionados à perda de peso no tratamento para obesidade. Esses autores demonstraram que idade, peso inicial, sexo e VCT foram associados à perda de peso. Além disso, nesta metanálise a redução ponderal foi inversamente associada à duração do estudo, achado também encontrado na presente amostra. Quanto ao uso de medicamentos antiobesidade, embora estejam associados a emagrecimento $(10,19)$, não observamos diferença de perda de peso entre os pacientes tratados ou não com medicação antiobesidade.

Entre as possíveis limitações deste estudo, está a não avaliação da adesão às intervenções prescritas (dietas e medicação). A adesão ao tratamento dietético é fator essencial para o sucesso de um tratamento para emagrecimento, independente do tipo de dieta prescrito (20). $\mathrm{O}$ delineamento adotado no presente estudo impediu que a adesão fosse avaliada, uma vez que na prática clínica dos consultórios não são utilizados instrumentos formais para tal.

Existem poucas medidas terapêuticas eficazes para o tratamento de sobrepeso e obesidade. Tratamentos tais como dietas, medicamentos e modificações de estilo de vida produzem modestas reduções no peso corporal, embora com impacto importante na saúde do indivíduo. No presente estudo, demonstramos que é possível perder peso de forma significativa - perda de peso média de $4 \mathrm{~kg}$, correspondendo a cerca de $5 \%$ do peso corporal inicial - em atendimento individualizado em consultórios privados. Ainda, o mais importante fator do sucesso terapêutico é um pequeno intervalo de tempo entre as consultas.

Declaração: os autores declaram não haver conflitos de interesse científico neste estudo.

\section{REFERÊNCIAS}

1. Ministério da Saúde, IBGE, Ministério do Planejamento, Orçamento e Gestão. Pesquisa de Orçamentos Familiares 2008-2009. Antropometria e estado nutricional de crianças, adolescentes e adultos no Brasil. Rio de Janeiro; 2010.
2. Ogden C, Carrol MD. Prevalence of overweight, obesity and extreme obesity among Adults: United States, Trends 1962 Through 2007-2008. CDC, NCHS downloaded from: http:// www.cdc.gov/nchs/data/hestat/obesity_adult_07_08/obesity_ adult_07_08.pdf

3. ABESO/SBEM. Atualização das diretrizes oficiais para tratamento farmacológico da obesidade e sobrepeso. Posicionamento Oficial da ABESO/SBEM; 2010.

4. Calle EE, Thun MJ, Petrelli JM, Rodriguez C, Heath CW Jr. Bodymass index and mortality in a prospective cohort of U.S. adults. N Engl J Med. 1999;341(15):1097-105.

5. Flegal KM, Graubard BI, Williamson DF, Gail MH. Excess deaths associated with underweight, overweight, and obesity. JAMA. 2005;293(15):1861-7.

6. American Diabetes Association: standards of medical care in diabetes, 2010. Diabetes Care. 2010;34:S11-61.

7. Chobanian AV, Bakris GL, Black HR, et al. The Seventh Report of the Joint Committee on Prevention, Detection, Evaluation, and Treatment of High Blood Pressure. JAMA. 2003;289:2560-72.

8. Sacks FM, Bray GA, Carey VJ, Smith SR, Ryan DH, Anton SD, et al. Comparison of weight-loss diets with different compositions of fat, protein, and carbohydrates. N Engl J Med. 2009;360(9):859-73.

9. Foster GD, Wyatt HR, Hill JO, Makris AP, Rosenbaum DL, Brill $C$, et al. Weight and metabolic outcomes after 2 years on a lowcarbohydrate versus low-fat diet: a randomized trial. Ann Intern Med. 2010;153(3):147-57.

10. Appel LJ, Clark JM, Yeh HC, Wang NY, Coughlin JW, Daumit G, et al. Comparative effectiveness of weight-loss interventions in clinical practice. N Engl J Med. 2011;365(21):1959-68.

11. Neovius M, Johansson K, Rossner S. Head-to-head studies evaluating efficacy of pharmaco-therapy for obesity: a systematic review and meta-analysis. Obes Rev. 2008;9(5):420-7.

12. Feigenbaum A, Pasternak S, Zusk E, Sarid M, Vinker S. Influence of intense multidisciplinary follow-up and orlistat on weight reduction in a primary care setting. BMC Fam Pract. 2005;6(1):5.

13. WaddenTA, Volger S, Sarwer DB, Vetter ML, Tsai AG, Berkowitz RI, et al. A two-year randomized trial of obesity treatment in primary care practice. N Engl J Med. 2011;365(21):1969-79.

14. Ashley JM, St Jeor ST, Schrage JP, Perumean-Chaney SE, Gilbertson MC, McCall NL, et al. Weight control in the physician's office. Arch Intern Med. 2001;161(13):1599-604.

15. Ahern $A L$, Olson $A D$, Aston LM, Jebb SA. Weight watchers on prescription: an observational study of weight change among adults referred to weight watchers by the NHS. BMC Public Health. 2011;11:434.

16. Willaing I, Ladelund $S$, Jorgensen $T$, Simonsen T, Nielsen LM. Nutritional counselling in primary health care: a randomized comparison of an intervention by general practitioner or dietician. Eur J Cardiovasc Prev Rehabil. 2004;11(6):513-2.

17. Franz MJ, VanWormer JJ, Crain AL, Boucher JL, Histon T, Caplan W, et al. Weight-loss outcomes: a systematic review and metaanalysis of weight-loss clinical trials with a minimum 1-year follow-up. J Am Diet Assoc. 2007;107(10):1755-67.

18. Finkler E, Heymsfield SB, St-Onge MP. Rate of weight loss can be predicted by patient characteristics and intervention strategies. $\mathrm{J}$ Am Diet Assoc. 2011 Oct 27.

19. Wadden TA, Berkowitz RI, Womble LG, Sarwer DB, Phelan S, Cato RK, et al. Randomized trial of lifestyle modification and pharmacotherapy for obesity. N Engl J Med. 2005;353(20):2111-20.

20. Alhassan S, Kim S, Bersamin A, King AC, Gardner CD. Dietary adherence and weight loss success among overweight women: results from the A TO $Z$ weight loss study. Int J Obes (Lond). 2008;32(6):985-91. 